VOL. 9 (1973), 29!-298.

\title{
A converse of Bernstein's \\ inequality for locally compact groups
}

\section{Walter R. Bloom}

Let $G$ be a Hausdorff locally compact abelian group, $\Gamma$ its character group. We shall prove that, if $S$ is a translationinvariant subspace of $L^{p}(G) \quad(p \in[1, \infty])$,

$$
\omega(a)=\sup \left\{\left\|\tau_{\alpha} f-f\right\|_{p}: f \in S,\|f\|_{p} \leq 1\right\}
$$

for each $a \in G$ and $\lim _{\alpha \rightarrow 0} \omega(\alpha)=0$, then $\underset{f \in S}{\bigcup} \Sigma(f)$ is

relatively compact (where $\Sigma(f)$ denotes the spectrum of $f$ ).

We also obtain a similar result when $G$ is a Hausdorff compact

(not necessarily abelian) group. These results can be

considered as a converse of Bernstein's inequality for locally

compact groups.

Throughout this paper we shall follow the notation of [1]. We require two technical lemmas.

LEMMA 1. Suppose we are given $x \in \Gamma$ and $k \in L^{1}(G)$ such that $\hat{k}(x)=1$. Then for $\varepsilon>0$, we can find $\imath \in L^{1}(G)$ such that $\hat{k} \hat{\imath}=1$ on a neighbourhood of $X$ and $\|l\|_{1}<1+\varepsilon$.

Proof. Choose $\delta \in(0,1)$ satisfying

$$
\delta(1-\delta)^{-1}<\varepsilon / 2
$$

Since $\left(\overline{\chi^{k}}\right)^{\wedge}(0)=1,[7]$, Chapter $5,2.3(5)$, p. 114, asserts the

Received 5 June 1973. Communicated by R.E. Edwards. The author would like to thank his supervisor, Professor Robert E. Edwards, for suggesting the problem. 
existence of $\tau \in L^{I}(G)$ such that $\|\tau\|_{1}<1+\varepsilon / 2, \hat{\tau}=1$ on a neighbourhood of zero, and

$$
\|(\bar{x} k) * \tau-\tau\|_{1}<\delta .
$$

Putting $\tau_{x}=x \tau$, (2) yields

$$
\left\|k * \tau x^{-\tau}\right\|_{1}<\delta,
$$

and clearly, $\hat{\tau}_{X}=1$ on a neighbourhood $V_{X}$ of $X$ and $\left\|\tau_{X}\right\|_{1}<1+\varepsilon / 2$.

As $\delta<1$, it appears from (3) that the series

$$
\tau_{x}+\sum_{n \geq 1}(-1)^{n}\left(k \star \tau x^{-\tau}\right)^{* n}
$$

converges in $L^{l}(G)$ to $Z$, say. For $\gamma \in V_{X}$, we have

$$
\begin{aligned}
\hat{k}(\gamma) \hat{z}(\gamma) & =\hat{k}(\gamma)\left(1+\sum_{n \geq 1}(-1)^{n}[\hat{k}(\gamma)-1]^{n}\right) \\
& =1 .
\end{aligned}
$$

A combination of (1), (3) and (4) gives us

$$
\begin{aligned}
\|z\|_{1} & \leq\left\|\tau_{\chi}\right\|_{1}+\sum_{n \geq 1} \delta^{n} \\
& <1+\varepsilon / 2+\delta(1-\delta)^{-1} \\
& <1+\varepsilon . \quad / /
\end{aligned}
$$

LEMMA 2. Let $\delta \in(0,1)$. Suppose that $X \in \Gamma$ and $a \in G$ satisfy

$$
|x(a)-1|>1-\delta
$$

Then we can find $p, q$ in $L^{l}(G)$ such that $\hat{p}=1$ on a neighbourhood of $X$,

$$
p=\tau_{a} q-q
$$

and

$$
\|q\|_{1}<(1-\delta)^{-1}(1+\delta) .
$$


Proof. By [8], 2.6.1, we can find $k \in L^{l}(G)$ such that $\hat{k}(x)=1$ and $\|k\|_{1}=1$. Since

$$
(\bar{x}(a)-1)^{-1}\left(\tau_{a}^{k-k}\right)^{\wedge}(x)=1
$$

we can appeal to Lemma 1 to deduce the existence of $Z \in L^{1}(G)$ such that $\|z\|_{1}<1+\delta$ and

$$
(\bar{\chi}(a)-1)^{-1}\left(\tau_{a} k-k\right)^{\wedge} \hat{\imath}=1
$$

on a neighbourhood of $x$. Now put

$$
q=(\bar{x}(a)-1)^{-1} k * 2
$$

Then, if

$$
p=\tau_{a} q-q
$$

(5) shows that $\hat{p}=1$ on a neighbourhood of $x$, and from (6),

$$
\begin{aligned}
\|q\|_{1} & \leq|\bar{x}(a)-1|^{-1}\|k\|_{1}\|z\|_{1} \\
& <(1-\delta)^{-1}(1+\delta) .
\end{aligned}
$$

We can now prove:

THEOREM 1. Suppose that $S$ is a translation-invariant subspace of ${ }_{L}^{p}(G) \quad(p \in[1, \infty])$, that

$$
\omega(a)=\sup \left\{\left\|\tau_{\alpha} f-f\right\|_{p}: f \in S,\|f\|_{p} \leq 1\right\}
$$

for each $a \in G$, and that $\lim _{a \rightarrow 0} \omega(a)=0$. Then $D=\underset{f \in S}{U} \Sigma(f)$ is relatively compact.

Proof. As $\omega$ is unchanged if we replace $S$ by $S^{-}$in (7), we can assume that $S$ is closed.

Suppose $D$ is not relatively compact. Then, if $V$ is any neighbourhood of zero and $\delta>0$ is given, we can find $a_{V} \in V, f_{V} \in S$ and $x_{V} \in \Sigma\left(f_{V}\right)$ such that

$$
\left|x_{V}\left(a_{V}\right)-1\right|>1-\delta
$$


(for if $|x(a)-1| \leq 1-\delta$ for all $a \in V$ and all $\chi \in D$, we could appeal to (23.16) of [6] to deduce that $D^{-}$is compact, contrary to assumption). In the case $p=\infty$, it follows from (7), the assumption that $\lim _{a \rightarrow 0} \omega(a)=0$, and the main resuit of [2] that $f_{V}$ is equal locally almost everywhere to a uniformly continuous function. Taking $\delta=1 / 4$, and recalling $(8)$, Lemma 2 implies the existence of an open neighbourhood $W_{V}$ of $x_{V}$, and $p_{V}, q_{V}$ in $L^{I}(G)$ such that $\hat{p}_{V}=1$ on $w_{V}$,

$$
p_{V}=\tau_{a_{V}} q_{V}-q_{V}
$$

and $\left\|q_{V}\right\|_{1}<2$.

Choose any $k_{V} \in L_{W_{V}}^{l}(G)$ such that $\hat{k}_{V}\left(\chi_{V}\right)=1$. Using the definitions of $p_{V}$ and $q_{V}$, we have

$$
\begin{aligned}
k_{V} * f_{V} & =p_{V} * k_{V} * f_{V} \\
& =\left(\tau a_{V} q_{V}-q_{V}\right) * k_{V} * f_{V} \\
& =q_{V} *\left(\tau a_{V} k_{V}-k_{V}\right) * f_{V} .
\end{aligned}
$$

Since $S$ is assumed to be a closed translation-invariant subspace of ${ }_{L}{ }^{p}(G)$, the proof of [7], Chapter $3,5.8$, p. 78 , can be used to show that

$$
h * f_{V} \in S
$$

for all $h \in L^{I}(G)$ (recall that when $p=\infty, f_{V}$ is equal locally almost everywhere to a uniformly continuous function). Combining (7), (9) and (10),

$$
\begin{aligned}
\left\|k_{V} \star f_{V}\right\|_{p} & \leq\left\|q_{V}\right\|_{I}\left\|{ }^{\tau} a_{V}{ }^{k_{V}}{ }^{\star f_{V}}-k_{V}{ }^{\star f_{V}}\right\|_{p} \\
& \leq 2 \omega\left(a_{V}\right)\left\|k_{V} f_{V}\right\|_{p} .
\end{aligned}
$$

As $x_{V} \in \Sigma\left(f_{V}\right)$ and $\hat{k}_{V}\left(x_{V}\right) \neq 0$, we see that $k_{V} * f_{V} \neq 0$ and so, by (11), 


$$
\omega\left(a_{V}\right) \geq 1 / 2
$$

Now consider the net $\left(a_{V}\right)$, where $V$ ranges over the set of neighbourhoods of zero, partially ordered by

$$
V \prec V^{\prime} \text { if and only if } V \supset V^{\prime} \text {. }
$$

It is seen that (13) entails that $\left(a_{V}\right)$ converges to zero; but (12) holds for all $V$, contradicting the assumption that $\underset{a \rightarrow 0}{\lim } \omega(a)=0$. Hence our assumption that $D$ is not relatively compact was false. //

REMARK. It can be shown that for the spaces $L^{\mathcal{I}}(G)$ and $C(G)$, we do not require that $\lim _{a \rightarrow 0} \omega(a)=0$ but only that there exists a compact set $F$ of strictly positive measure such that $\omega(a)<\alpha<1$ for all $a \in F$.

COROLLARY 1. Let $M_{b}(G)$ denote the space of bounded Radon measures on $G$. Suppose that $S$ is a transzation-invariant subspace of $M_{b}(G)$, that

$$
\omega(a)=\sup \left\{\left\|\tau_{a} \mu-\mu\right\|_{M}: \mu \in S,\|\mu\|_{M} \leq 1\right\}
$$

for each $a \in G$, and that $\lim _{a \rightarrow 0} \omega(a)=0$. Then $\underset{\mu \in S}{U}$ supph is relatively compact.

Proof. It follows from (14) and [3], Corollary 3, that any $\mu \in S$ is generated by an $L^{1}$-function. Let

$$
S^{\prime}=\left\{f \in L^{1}(G): f \text { generates a measure in } S\right\} .
$$

Then $S^{\prime}$ is a translation-invariant-subspace of $L^{l}(G)$ satisfying the conditions of Theorem 1 , from which we deduce that $\underset{f \in S^{\prime}}{U} \Sigma(f)$ is relatively compact. Since $\hat{f}=\hat{\mu}_{f}$, where $\mu_{f}$ is the measure generated by $f$, and any $\mu \in S$ is $\mu_{f}$ for some $f \in S^{\prime}$, we can conclude (note that

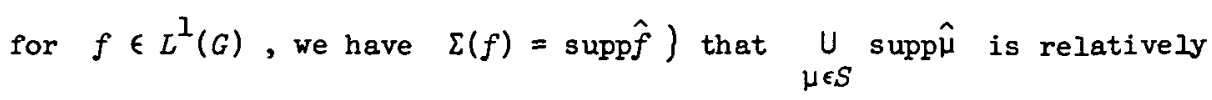
compact. // 
We shall now consider the converse when $G$ is a Hausdorff compact group ( $G$ is not assumed to be abelian). We follow the notation used in [5]. Given a finite-dimensional continuous irreducible unitary representation $U \in \hat{G}$, with representation space $H_{U}, d(U)$ will denote the dimension of $H_{U}$, and $I_{U}$ the identity endomorphism of $H_{U}$. The trace function on $H_{U}$ will be denoted by $T r$. We let $(E(G),\|\cdot\|)$ denote any of the spaces $I^{P}(G) \quad(p \in[1, \infty))$ or $C(G)$, each taken with its usual norm. By $L_{a}$, we will mean the left translation operator.

THEOREM 2. Suppose that $S$ is a left trans Lation-invariant subspace of $E(G)$, that

$$
\omega(\alpha)=\sup \left\{\left\|L_{\alpha} f-f\right\|: f \in S,\|f\| \leq 1\right\}
$$

for each $a \in G$, and that $\lim _{a \rightarrow 0} \omega(a)=0$. Then $\underset{f \in S}{U}$ supp $\hat{f}$ is finite.

Proof. As $\omega$ is unchanged if we replace $S$ by $S^{-}$in (15), we can assume that $S$ is closed.

Consider the unit disc in $S$;

$$
B=\{f \in S:\|f\| \leq 1\} .
$$

It follows immediately from the Weil criterion ([4], 4.20.1), or when $E(G)=C(G)$, from Ascoli's Theorem ([4], 0.4.11), that $B$ is compact in $E(G)$. We can now use the Riesz Theorem ([4], p. 65) to deduce that $S$ is finite dimensional.

Let $\left\{f_{1}, f_{2}, \ldots, f_{n}\right\}$ be a basis for $S$. Since for every $f \in S$,

$$
\text { supp } \hat{f} \subseteq \bigcup_{j=1}^{n} \operatorname{supp}_{j},
$$

it will suffice to show that $\operatorname{supp}_{j}$ is finite for all $j \in\{1,2, \ldots, n\}$.

However if this were false, there would exist $j \in\{1,2, \ldots, n\}$ and an infinite sequence $\left\{U_{i}\right\}_{i=1}^{\infty}$ of distinct elements of $\hat{G}$ such that $\hat{f}_{j}\left(U_{i}\right) \neq 0$ for every $i \in\{1,2, \ldots\}$. Define $h_{i} \in C(G)$ by

$$
h_{i}(x)=d\left(U_{i}\right) \operatorname{Tr}\left[U_{i}(x)^{*}\right] \text {, }
$$


where $U_{i}(x)^{*}$ denotes the adjoint of $U_{i}(x)$. Since $S$ is assumed to be a closed left translation-invariant subspace of $E(G)$, it is a left ideal (in $E(G)$ ); hence $h_{i} * f_{j} \in S$ for every $i \in\{1,2, \ldots\}$. Also

$$
\begin{aligned}
\left(h_{i} * f_{j}\right)^{\wedge}\left(U_{k}\right) & =\hat{h}_{i}\left(U_{k}\right) \hat{f}_{j}\left(U_{k}\right) \\
& =\delta_{i k} \hat{f}_{j}\left(U_{k}\right),
\end{aligned}
$$

where

$$
\delta_{i k}= \begin{cases}I_{U_{k}}, & i=k, \\ 0, & i \neq k .\end{cases}
$$

We see that $\left\{h_{i} * f_{j}\right\}_{i=1}^{\infty}$ is linearly independent in $S$; for suppose there exist $\alpha_{i} \in C$ such that

$$
\sum_{i=1}^{m} \alpha_{i}\left(h_{i}{ }^{\star} f_{j}\right)=0 .
$$

Then for all $k$,

$$
\sum_{i=1}^{m} \alpha_{i}\left(h_{i} * f_{j}\right)^{\wedge}\left(U_{k}\right)=0
$$

and by (16),

$$
\sum_{i=1}^{m} \alpha_{i} \delta_{i k} \hat{f}_{j}\left(U_{k}\right)=0
$$

that is,

$$
\alpha_{k} I v_{k} \hat{f}_{j}\left(U_{k}\right)=0
$$

Since $\hat{f}_{j}\left(U_{k}\right) \neq 0$, it follows that $\alpha_{k}=0$ for all $k$. Hence $\left\{h_{i} \star f_{j}\right\}_{i=1}^{\infty}$ is linearly independent in $S$, contradicting the fact that $S$ is finite dimensional. Consequently supp $\hat{f}_{j}$ is finite for all $j \in\{l, 2, \ldots, n\}$, and the
theorem is proved. $/ /$

COROLLARY 2. Suppose that $S$ is a left translation-invariant subspace of $L^{\infty}(G)$, that

$$
\omega(a)=\sup \left\{\left\|L_{a} f-f\right\|_{\infty}: f \in S,\|f\|_{\infty} \leq 1\right\}
$$

for each $a \in G$, and that $\underset{a \rightarrow 0}{\lim } \omega(a)=0$. Then $\underset{f \in S}{U}$ supp $\hat{f}$ is finite. 
Walter R. Bloom

Proof. It follows from (17.) and the proof of the main result of [2] that every $f \in S$ is equal almost everywhere to a uniformly continuous function. The problem is then reducible to that covered by the case $E(G)=C(G)$ of Theorem 2 . //

\section{References}

[1] Walter R. Bloom, "Bernstein's inequality for locally compact abelian groups", J. Austral. Math. Soc. (to appear).

[2] D.A. Edwards, "On translates of $L^{\infty}$-functions", J. London Math. Soc. 36 (1961), 431-432.

[3] R.E. Edwards, "Translates of $L^{\infty}$ functions and of bounded measures", J. Austral. Math. Soc. 4 (1964), 403-409.

[4] R.E. Edwards, Functional analysis: Theory and applications (Holt, Rinehart and Winston, New York, Chicago, San Francisco, Toronto, London, 1965).

[5] R.E. Edwards, Integration and harmonic analysis on compact groups (Notes on Pure Mathematics, 5. Australian National University, Canberra, 1970).

[6] Edwin Hewitt and Kenneth A. Ross, Abstract harmonic analysis, Volzome I (Die Grundlehren der mathematischen Wissenschaften, Band 115. Academic Press, New York; Springer-Verlag, Berlin, Göttingen, Heidelberg; 1963).

[7] Hans Reiter, Classical harmonic analysis and locally compact groups (Clarendon Press, Oxford, 1968).

[8] Walter Rudin, Fourier analysis on groups (Interscience, New York, London, 1962; 2nd Printing, 1967).

Department of Mathematics, Institute of Advanced Studies, Australian National University,

Canberra,

ACT. 